\title{
Mother-Daughter Dyad Recruitment and Cancer Intervention Challenges in an African American Sample
}

\author{
Maghboeba Mosavel • Katie A. Ports • \\ Ellyn Leighton-Herrmann
}

Received: 14 January 2014 /Revised: 24 March 2014 / Accepted: 30 April 2014 /Published online: 23 May 2014

(C) W. Montague Cobb-NMA Health Institute 2014

\begin{abstract}
Developing an effective youth-based health messaging intervention for African American women, who remain disparately impacted by cancer, presents unique challenges. This paper reports on the challenges with recruiting familial dyads from predominantly low-income, African American neighborhoods, as well as the challenges associated with designing and implementing an upward-directed cancerscreening intervention. We developed and pilot-tested an educational workshop that provided adolescents with cancerscreening information to share with their mother or female relative. Data from follow-up interviews and focus groups, as well as observation records regarding implementation obstacles, highlight important lessons learned. The use of familial dyads as well as issues of access posed challenges for recruitment and follow-up. Workshop-related challenges stemmed from the approach, content, and length. Finally, personal and environmental factors presented barriers to adolescent message delivery and adult follow-through. By identifying these challenges, we hope to influence and enable the future development of effective adolescent-initiated health-messaging interventions.
\end{abstract}

Keywords Mother-daughter dyads · Cancer-screening intervention $\cdot$ Recruitment challenges $\cdot$ African American

\section{Introduction}

Despite increased availability of screening resources, tremendous research and targeted education efforts, cancer deaths

M. Mosavel $(\square) \cdot$ K. A. Ports $(\square) \cdot$ E. Leighton-Herrmann Department of Social and Behavioral Health, School of Medicine, Virginia Commonwealth University, PO Box 980149, Richmond, VA 23219, USA

e-mail: mmosavel@vcu.edu

e-mail:kaports@vcu.edu continue to disproportionately affect African American women in the USA[1-3]. Insufficient knowledge about cancerscreening guidelines and tests is a significant barrier to participation in preventive cancer screening, especially among minority populations, which contributes to disparate cancer mortality rates [4-7]. African American women are more likely to be diagnosed at later stages of the disease $[8,9]$ and thus have significantly higher mortality rates from cancer $[2,3,10]$. Furthermore, perceived barriers to screening vary across levels of socioeconomic status (SES) as well as within racial groups, with minority and lower-income women having higher levels of perceived and actual barriers to cancer screenings [11]. Persistent disparities in cancer diagnosis and mortality, as well as limited cancer knowledge highlight the need for additional research aimed at developing culturally tailored and relevant cancer-screening interventions.

Research that is both sensitive to cultural and social contexts is critical to efforts to reduce existing cancer disparities. Unfortunately, populations that would benefit most from health disparities research remain underrepresented in research and are less likely to participate in such research for myriad reasons [12-15]. Researchers' ability to assess and address health disparities within racial and ethnic minority populations, as well as differences between groups is subsequently hindered by the limited participation of these harderto-reach populations $[16,17]$. Increasing the participation of minority populations would allow for ethnicity-specific analyses, which would aid in the development of culturally tailored interventions targeted at the populations most in need [16].

Recent cancer interventions have demonstrated promise for the inclusion of family communication in targeted cancer education efforts $[18,19]$. More specifically, the use of upward communication (i.e., communication from child to adult) has been identified as a potential channel for the delivery of health information within minority families $[20,21]$. However, young people are often overlooked as a resource for 
health promotion within the family system including the extended family. As such, there remains a paucity of resources about upward and bidirectional health promotion messages within the family network.

The expanding opportunities for, and access to, new information puts adolescent females in a position to inform and influence their mother or female relative's cancer-screening behavior [22]. In the African American community, women are most likely to be the single head of household (27.5\%), in comparison to White $(9.4 \%)$ and Asian $(7.5 \%)$ women who are least likely [23]. Having a single-mother head of household puts the youth within the family in an important position to help out and even provide important information to the mother. Existing literature suggests that mothers are more likely to talk with their children about a larger variety of personal health topics, compared to fathers [24-26]. Because the mother-daughter bond is an especially strong and stable relationship [27], daughters may be a particularly important source for communicating health messages to the mother. Indeed, previous research in mother-daughter communication indicates that African American adolescent girls are able and willing to share health information with their mother and that the mother is also willing and eager to receive the information from her daughter [22, 28].

Developing effective health messages for youth to deliver to their parent or adult relative may present unique challenges. Researchers recommend that health interventions targeting youth should aim to establish group identity, allow time for socializing, use creative approaches to disseminate content and group facilitators that are relatable [29, 30]. While informative, we know little about developing specific, normative cancer-screening messages that can be effectively disseminated in an upward-directed manner-from adolescent to adult.

Beyond the design of an appropriate intervention message, researchers and practitioners also need to understand the different challenges associated with dyadic interventions, which may compound well-documented challenges with recruitment of minorities [14, 15, 31-33]. While dyadic research has been used in several settings, including community and public health settings [34], much less is known about the specific challenges related to the recruitment and retention of adultadolescent familial dyads especially among those who are low income, underserved, and underresourced. Some general challenges that have been reported in dyadic research include needing both members of the dyad to meet inclusion criteria or be willing to participate $[31,32]$. When conducting research with the African American family, the inclusion of the extended family is an important consideration. Extended family members are an integral component of the African American familial network [35] and research suggests that extended familial relationships are more salient for African Americans [36]. More information is needed on how to better recruit and retain dyads, including extended family members, especially in populations disparately impacted by cancers.

This paper reports on the lessons we learned related to recruitment and designing an upward-directed, adolescent to mother-relative intervention implemented among African Americans women who lived in low-income neighborhoods and were noncompliant with either breast, cervical, or colon cancer-screening recommendations. Information about the lessons we learned and how we were able to adapt our daughter-initiated intervention to the challenges presented by the real world, on the ground realities, may be useful to others who work with similar populations and who may experience barriers recruiting and retaining participants, especially dyads from low-income communities.

\section{Study Overview}

A community-based study was conducted to examine the feasibility of girls, ages $12-18$, providing their mothers or female relatives with either breast, cervical, and/or colon cancer-screening information, with the goal of persuading them to obtain the screening they need. Girls in the intervention group were exposed to an educational workshop, while those in the control group were provided with a pamphlet with information on the importance of screening and the specific steps they can take to help their mother/relative obtain screening. In this paper, we first provide information about the development and pilot testing of the workshop. We then discuss the implementation of the pilot workshop as first designed and the subsequent changes that were made to the design. Next, we discuss the implementation of the workshop in the feasibility study, and we specifically explore the various issues we experienced with recruitment of this specific population. Finally, we discuss the overall results of the intervention as well as additional lessons we learned even after implementing the feasibility study.

\section{Methods}

Sample

The sample included five dyads $(n=10)$ for the pilot testing phase and 48 dyads $(n=96)$ for the feasibility study, of which 30 were assigned to the intervention group and 18 to the control group. Eligibility criteria included adult women 40 years or older, who reported being uninsured or underinsured and not compliant with screening recommendations for either breast, cervical, or colon cancer. Women with a previous cancer diagnoses were excluded. The screening recommendations at the time of study recruitment recommended that women receive a yearly mammogram, a Pap smear once 
every 24 months, and if 50 or older, a yearly colorectal exam. To be eligible, the adolescent needed to be between the ages of 12 and 18 and in grades 6 through 12. Exclusion criteria for the adolescents included being pregnant, having a child or not having been in school in the past 6 months. Eligible dyads included mother-daughter pairs, or if the mother was not eligible, a female relative.

\section{Recruitment}

Given that the focus of this research is to decrease cancer disparities, especially cancer mortality rates of African American women, we focused recruitment efforts on African American participants who resided in urban, low-income neighborhoods including subsidized housing communities. Participants were recruited from various sites including recreation centers, community centers, churches, after school programs, and adult-centered programs.

We used a group-randomized design where neighborhood centers were the units of assignment and the units of observation were the dyads. For example, all participants recruited from neighborhood A and locations within close proximity to that neighborhood were assigned to the same condition. In most cases, the mother was the initial point of contact for recruitment; however, in at least $25 \%$ of cases, the adolescent was the first contact. The majority of mothers reported hearing about the study from the church, community center, or outreach worker or project staff $(n=24,67 \%)$. Conversely, most daughters heard about the study from their mother, grandmother, or aunt with whom they participated $(n=27,75 \%)$. If the adolescent was the first person to be reached, after ascertaining initial interest, she either contacted her mother immediately or took informational material home. If mothers were the first point of contact, we would ask them about the eligibility criteria for both themselves and their daughter. In cases where a woman was ineligible, we asked her to identify a relative who might be eligible. About $55 \%$ of intervention dyads and $64 \%$ of control dyads were mother-daughter pairs. The remaining dyads comprised an adolescent and an adult female kin (e.g., grandmother).

\section{Informed Consent}

We received ethics approval to conduct the study and we submitted and received approval for various protocol changes to accommodate the lessons from the field. Once the dyad was determined eligible, we arranged to conduct informed consent, usually at that time of the baseline interview. All adolescents were asked separately about their willingness to participate and they completed written assent. In cases where the adolescent was not participating with her mother, we obtained permission and consent from her mother, before conducting consent with the eligible adult female relative. The consent form indicated that the study was a health communication project, which examined communication between adolescent and mother or relative. Given the need to provide the daughter with information tailored to the adult relative's screening needs, we asked the adults during their baseline interview if the researcher could share their screening information with the adolescent. We also requested permission to access their medical records, with the stipulation that medical records would only be accessed if they received services from one of the clinics affiliated with the researchers' institution. All of the women consented. In the group of five pilot dyads, both the adults and adolescents received $\$ 60$ for their participation. Adults in the feasibility study, both the intervention and control groups, received $\$ 30$ at baseline and $\$ 50$ at exit. Adolescents received a total of $\$ 65$.

\section{Process}

Of the five dyads, four attended the pilot workshop. We pilottested the workshop content and design with the goal of modifying the workshop for the feasibility study. Additionally, we wanted to assess the intervention design particularly as it related to girls being able to deliver the cancer message and appeal to the intended recipients in their home setting. In previous work (Mosavel and Wilson, under review), the message itself was tested in an observational setting, but we lacked information about the message delivery in the home setting. Two weeks after the workshop, we followed up and conducted a group discussion with the girls and individual interviews with the adults.

For the feasibility study, those assigned to the intervention group attended a workshop scheduled within 7 days to a month after first completing the baseline interview. Both control and intervention dyads were followed up within 36 months later (the broad range in follow-up time was due to scheduling issues). Community recruiters were responsible for recruiting and, in some cases, administering the interviews. A trained researcher, who was female and African American, facilitated workshops.

\section{Workshop Content}

The purpose of the workshop was to provide adolescents information about cancer disparities as it pertained to African Americans, highlighting the lifesaving importance of early detection and, in particular, the need for their mother or relative to be screened. The elaboration likelihood model (ELM) holds that interventions that utilize the central route to persuasion by involving deeper thinking about a message will result in a more persuasive appeal and thus will increase the likelihood of the desired behavior [37]. While the emotional connection or bond with the mother or relative was deemed to 
be an important aspect of internalizing the cancer message [37], this pilot workshop focused primarily on racial disparities because mothers were very receptive to this message in previous work (Mosavel and Wilson, under review). Based on the previous research [37] and directed by the elaboration likelihood model (ELM) [38], the overarching goal of the workshop was to focus on cancer disparities to prompt the girls to understand the importance of their mothers or relatives being vigilant about the primary prevention of cancer, thus internalizing the message, resulting in the development of a persuasive appeal for their elder family member to participate in cancer screening.

Because mothers were receptive to information regarding racial disparities in previous work, the workshop content was presented within an Afrocentric empowerment framework [39] that attempted to communicate the historical legacy and significant contributions of African Americans. The workshop facilitator, an African American woman, wove sociocultural information into the workshop to provide a context that tied the relevance of historical legacy to cancer disparities in the African American community. The workshop included a PowerPoint presentation that highlighted cancer incidence and mortality data [1-3] as well as three YouTube videos of personal testimonials from African Americans about the importance of breast, cervical, and colon cancer screening. One of the YouTube videos starred actor Terrence Howard who provided an emotional testimonial about his mother's colon cancer diagnosis. Pre- and post-tests assessed adolescents' cancer knowledge and immediate recall of workshop information.

According to the theory of planned behavior, behavioral intentions strongly predict behavior in which individuals can exert control; intentions are influenced by attitudes about the likelihood that the behavior will have the expected outcome and the subjective evaluation of the risks and benefits of that outcome [40]. Using the theory of planned behavior [40], the girls were asked to develop a plan for their message delivery, which was called the "My Plan" and included a personalized persuasive message that was applicable to their relationship and family situation, about obtaining the necessary screening test. In order to increase intention, adolescents were provided with an envelope containing information about specific screening(s) that their relative needed. In their My Plan, they identified when they would share the message, what they would say, and the tone with which they would deliver the message and make the cancer-screening appeal. They also had the opportunity to brainstorm and role-play with other group members to enhance their sense of behavioral control.

Before concluding the workshop, girls were given a pamphlet to take home. The title was "What would YOU do if your loved one got cancer? What your relative should know. What YOU can do to help. A guide for youth.” This pamphlet outlined six steps they could take to help their relative get screened for cancer. It also contained a list of local and other resources for screening. Finally, girls were asked to text the project coordinator after they delivered the cancer message to their female kin.

\section{Data Sources and Analysis}

Data for this paper include information collected during the pilot testing of the workshop as well as the implementation of the feasibility study.

\section{Data Sources from the Pilot Testing}

Detailed notes were taken by the research assistants and observations included nonverbal responses, flow of content, attention span and interest, and comprehension of materials. Two weeks later, in a group setting, the adolescents were asked questions about recall of the cancer-screening information and implementing their My Plan. Follow-up interviews were conducted with the adult relatives. They were asked what, if any, information the adolescent shared with them about the workshop. They were also asked specifically whether they received the cancer-screening appeal.

\section{Data Sources from the Feasibility Study}

The project coordinator, community researchers and research assistants submitted process notes focusing on issues related to recruitment and implementation. Observational data about participants' comprehension and engagement were also recorded and post-workshop evaluations were completed.

\section{Results}

\section{Pilot Workshop Results}

This next section reports the findings about the pilot workshop and is followed by a discussion of the subsequent changes made to the workshop. Based on multiple sources of feedback, we established that having the girls complete a My Plan was helpful in directing them to a feasible plan for message delivery. The My Plan helped them identify obstacles in terms of time and/or developing the wording for their cancer-screening appeal. The participants also found the envelope containing specific cancer-screening information very enlightening, because this information was specific to their relative and made the need for screening appear very relevant and even urgent. All of the girls reported that the role-playing activities were very helpful in preparing to deliver the cancer appeal. Finally, the adolescents found the pamphlet to be useful to share with their relatives and provided them with additional support to 
their extemporaneous message. Below, we discuss elements of the workshop that were perceived as less effective.

\section{Message Sharing}

During the follow-up group discussion with the adolescents, we assessed what did and did not work regarding the message sharing process. Based on this discussion, we found that of the four adolescents who participated in the pilot workshop, three shared their message with their relative, and one shared her message with a relative who was not enrolled in the study. When asked why, she said: "Because my grandmother was not here."

None of the adolescents remembered to text the project coordinator. When asked why, they said that they forgot to do so and that they became busy with their life. In response to how they executed their My Plan, responses included, "I did not text $N$ [project coordinator], but I did talk to my mother about what we talked about," and, "What I did was I told my mom after I got home from the workshop and after I told her, she never went to the doctor or nothing and I told her again and she still didn't do it." Follow-up interviews with relatives indicated that while the daughter shared the information, she did not specifically ask her adult female kin to be screened. All of the adults said that the youth gave them the pamphlet.

\section{Workshop Focus}

Based on participants' responses during the workshop, they struggled with thinking about significant historical events in relation to their culture and were more likely to refer to contemporary popular culture. During the workshop, we observed that the adolescents were able to make a strong emotional connection when asked to discuss how they would feel if they were to lose their relative with whom they were participating in the study with. Further, the emotionally focused aspects of the workshop seemed to resonate more strongly with the adolescents. In addition, when we assessed recall about the workshop, the emotional testimony presented in the YouTube video with the actor Terrance Howard appeared to have made an impression on the adolescents. In general, they were better able to recall information presented within an emotional context compared to the more matter-offact information.

\section{Workshop Content and Duration}

Based on observations made during the workshop as well as the debriefing reports, the girls started to lose focus due to the 2-h duration of the first workshop. The follow-up discussion with the adolescents confirmed that the workshop was very long and the girls had difficulty remembering much of the PowerPoint information.
Lessons Learned and Changes Made to the Workshop

\section{Workshop Focus}

The Afrocentric focus did not resonate with these adolescent participants. However, what did seem to capture their attention was the emotional connection with their relative and the personal meaning of this person in their life. As such, we revised the focus of the workshop and underscored the girls' personal relationship with her mother or adult relative.

\section{Less Is More-Repeat Often}

Observations recorded during the pilot workshop suggested that the adolescents were having difficulties comprehending all the information and that they had, sometimes vastly, differing cognitive abilities. As a result, we reduced the overall time of the workshop from 120 to $90 \mathrm{~min}$. More importantly, we shortened and simplified the information presented in the PowerPoint by focusing the screening information around a moniker that we developed in response to girls having difficulty understanding the concept of early detection for cancer. The moniker was captured by the introduction of the " $3 \mathrm{Gs}$ " which highlighted the need to (1) Go and get screened early, (2) Go for screening regularly, and (3) Go for screening follow-ups, if needed. The revised presentation contained fewer slides, presented basic screening test information about the three cancers, and focused on the three main concepts of the 3Gs. During the role play, we highlighted the importance of the adolescent going beyond sharing the information and the need to specifically ask or appeal to their relative to obtain the necessary screen(s).

\section{Reminders}

Given that the participants neglected to text the project coordinator, during the follow-up discussion with adolescents, we sought suggestions about what might motivate them to text once they delivered the message. They indicated the need for reminders and shared how busy and, at times chaotic, their lives are so that sending a text about a project is not something that they would easily remember. Furthermore, based on new insights about the differing schedules of the adolescent and adult relative, we strengthened the My Plan by having the youth more deliberately think through her plan as to when she could realistically deliver the message to the intended recipient.

\section{Implementation of Workshop}

While the revised workshop curriculum followed a format similar to that of the pilot curriculum, we made substantial changes to the focus of the workshop (from Afrocentric to 
emotional bond with relative), shortened the workshop from $2 \mathrm{~h}$ to $90 \mathrm{~min}$, reduced and simplified the content, and developed the screening message around the 3Gs. We also added the 3 Gs to the pamphlet that all adolescents received and retitled it, "The 3Gs of Cancer Screening. What your relative should know. What YOU can do to help. A guide for youth." We also provided the girls with a "Next Steps" card the size of a business card, which included the project coordinator's cell phone number and a reminder to text the project coordinator. Finally, for the feasibility study, we provided intervention arm girls with a journal and asked her to record the conversation she had with her relative about screening. Specifically, we asked the girls to describe how the conversation went, what some of the challenges were, and how she felt during and after the conversation. We asked the adolescents to bring their journals with them to the exit interview.

Once the workshop was implemented for the feasibility study, we realized that 90 min was still very lengthy. We further reduced the time to $60 \mathrm{~min}$, which appeared to be the ideal time frame for the girls to remain engaged while sharing sufficient information and conduct roleplays and the My Plan. Finally, we included the following incentive: girls who text the coordinator within 1 week of the workshop will be entered into a raffle for $\$ 100$.

\section{Recruitment Challenges}

For the feasibility study, at baseline, we enrolled 30 dyads $(n=60)$ who were assigned to the intervention and 18 dyads $(n=36)$ were assigned to the control arm. Attrition was at $26 \%$ in the intervention arm; three adolescents completed their exit interview on the telephone (however, they are not included in the final dataset). Given that this was a feasibility study, we intentionally over recruited for the intervention group. In the control arm, attrition was at $22 \%$. Due to scheduling problems, we closed the recruitment of the control group before we reached the preferred recruitment number of 20 dyads per group.

Recruitment, retention, and follow-up for this study presented various challenges that necessarily influenced the implementation. Many of the challenges are inevitable since the study participants were from a high-risk group (lack of screening) as well as from a sample with high unemployment with incomes well below the national poverty guidelines. Slightly more than one third of the sample $(n=13,36 \%)$ reported making less than $\$ 5,000$ a year and 11 (31\%) reported between $\$ 5,000$ and $\$ 20,000$. Six $(17 \%)$ were uninsured and $23(64 \%)$ had some form of subsidized health coverage.

Recruitment challenges were confounded by the specific eligibility criteria both for individuals and the dyad. Challenges arose when one potential dyad member was willing to participate, but their possible counterpart was unwilling, ineligible, or unavailable. The dyad-based approach also complicated individual scheduling since we required both participants to be together for the baseline and follow-up interviews. Identifying and recruiting eligible dyads required considerable time. We utilized two community members as recruiters. The community recruiters indicated that they had difficulties with the set time lines for enrollment and that they experienced major delays scheduling. In some cases, the eligible adult told the community recruiter that the youth met the eligibility criteria for grade or age. However, when the youth was assented by a member of the research team, her eligibility did not match.

\section{Challenges with Scheduling and Follow-Up}

Scheduling of, and follow-up with, the recruited pairs was made more challenging due to changing phone numbers and disconnected phone lines. When follow-up interviews were scheduled 3 months later, often the multiple phone numbers provided at baseline were not functioning. Although the majority of the adolescents had a cell phone (86\%), we learned that most had a cell phone with limited minutes. At follow-up, some of the intervention arm girls said they were unable to text, even though they delivered the message, because they did not have minutes left and when they received their new allocation of minutes was restored later on, they would forget to text to the coordinator. To help with workshop attendance, we conducted the workshop in the neighborhood where the adolescent was recruited and in some cases, we provided transportation. However, in some cases, transportation was still an issue often because plans for transportation would either not materialize or some unforeseen issue arose.

\section{Sharing the Screening Appeal}

In the intervention group, $86 \%(n=19)$ of the adolescents shared the screening message with their adult female relative and $82 \%$ of adults $(n=18)$ reported having received the screening appeal. Forty-two percent of adults $(n=13)$ reported they received the pamphlet. Fourteen (74\%) adolescents reported that the message was shared the same day as the workshop. Of the girls who shared the message, $74 \%(n=14)$ sent a text message to the coordinator to say they shared the screening appeal. All the women who reported receiving the message said that they were specifically asked to get screened. After the first conversation, $74 \%(n=14)$ of adolescents said they followed up with their relative, and $67 \%$ (12) adults recalled that the adolescent followed up with her. In addition, $74 \%(n=14)$ said they told their female relative about the $3 \mathrm{Gs}$. However, at follow-up, only two (11\%) of the 19 girls who shared the message said they remembered what the $3 \mathrm{Gs}$ are, and only one named all three.

In the control group, $71 \%(n=10)$ of both the adolescents and adults reported that the youth shared the cancer message. 
Five $(50 \%)$ adolescents and three $(30 \%)$ adults reported that the message was shared the same day. Nine $(64 \%)$ of the adolescents reported sharing the pamphlet of information with their adult relative, while $42 \%$ of adults $(n=6)$ reported having received the pamphlet of information. Six (43\%) women who reported receiving the message said that they were specifically asked by the adolescent to get screened for cancer. However, only two adolescents reported that they (14\%) were specifically asking their adult relative to get screened. After the first conversation, $20 \%(n=2)$ of adolescents said they followed up with their relative and $30 \%$ (3) adults recalled being follow-up with.

For the girls who did not share the message, regardless of study arm, the majority of reasons centered on being very busy, not remembering, and not being able to find the right time. Some of the comments included "Time - and I had other things on my mind - school, testing and she had things to do, too. Timing, it was never the right time and then it would slip my mind and I would forget." And, "Because I had like a whole bunch of things and forgot about it, but I read it [the pamphlet]." Or "Nothing at home prevented me. It just skipped my mind. Each time (I) thought about it, I put it off talking to her. I was not afraid or uncomfortable talking to my mother. I just forgot."

\section{Discussion}

This paper reports on the challenges associated with recruiting familial dyads from mostly poor, underserved, predominantly low-income neighborhoods and the accompanying issues related to designing and implementing an upward-directed cancer-screening message for adolescents to share with their mothers or female relatives. Involving African American women in cancer intervention research is a rich source for informing culturally relevant health experiences and concerns and may provide opportunities to address existing cancer disparities. To date, few studies have investigated the use of daughter-initiated cancer interventions or the challenges specifically associated with the recruitment of mother-daughter dyads and development of a tailored cancer intervention. The women and adolescents in this study provided a generous view into their lived experiences and demonstrated how interventions must be developed in a real world context to have relevance and impact.

We found that adolescents who participated in an informational cancer workshop were able to share a cancer message with their adult relative including making an appeal for the adult to participate in cancer screenings. Although the majority of girls in the control arm shared the cancer information with their female kin, they were less likely to make a screening appeal, and less likely to follow up with their kin about cancer screening. Despite the promising outcomes from the intervention, adolescents struggled to remember the content of the workshops and, at times, forgot to follow through with their study plan. We found that providing a short moniker, the 3Gs, placing emphasis on the girls' personal connection to the adult female and shortening the workshop resulted in better retention of the information.

Previous research has suggested that incorporating Afrocentric messages into interventions targeting African Americans can be effective [41, 42]; however, the adolescent females in this study struggled to place the relevance of cancer disparities in the context of historical contributions and struggles of African Americans. Rather, we found that highlighting the connection between the adolescent and her relative was most effective in being a motivator to share cancer and screening information. The elaboration likelihood model (ELM) suggests that some communication messages are processed differently depending on saliency or involvement with the topic [43], because the Afrocentric message was less relevant than the personal connection, we shifted the content of the workshop to reflect this. However, it is important not to dismiss the value of an Afrocentric approach since most programs utilizing this approach did so over several weeks $[30,44]$. Attempting to incorporate an Afrocentric approach within one session, as was the case in our study, is likely to be ineffective. The content of the workshop and the focus on the emotional bond can have relevance for all mothers and daughters across the spectrum since it capitalizes on a universal emotion well beyond race and ethnicity. Of future interest is the larger, community impact of this intervention. Future research should examine if participants share the cancer information with others in their social networks.

Beyond the challenges associated with the content of the workshops, we also experienced challenges with recruiting and retaining participants. Recruitment challenges with minority participants in research have been well documented $[16,17]$. Because the feasibility study was a communitybased intervention, we utilized community recruiters to attempt to reduce recruitment challenges. Given their lay position, community recruiters are highly effective at recruitment of especially hard to reach populations. However, this recruitment and access method does not come without challenges to the scientific process [45]. In this study, these challenges manifested around issues of recruitment and eligibility. It is important to consider the amount of time and energy that goes into recruiting, as well as the cultural context of family relationships within poor, low-income, minority populations. We found that while the research team was open to including dyads that were not traditional mother-daughter relationships, participants were weary of disclosing relationship status and may have benefited from more time/exposure with the community recruiter.

The use of dyads presented specific challenges to retention in this study. Scheduling dyads to participate in interviews at 
the same time was difficult, because the adults and the adolescent often had conflicting schedules. We also faced challenges with scheduling participants for workshops. The time period between baseline and follow-up interviews varied from 3 to 6 months, largely due to issues with making initial contact with the adult. In addition, adolescents were supposed to attend the workshop within 1 week of their baseline interview. However, in some cases, it took up to a month to schedule a workshop that five to six adolescents could all attend. Due to scheduling conflicts, we closed the recruitment of the control group dyads before we reached the desired number, resulting in an uneven number between the intervention and control groups.

In an intervention study focusing on high-risk, African American families from low-income inner-city communities, researchers enhanced retention by promoting participants' sense of anticipation, progress, and achievement as well as by reducing potential barriers to attendance [46]. In this study, we also attempted to address barriers to attendance. We strategically held workshops within the community and provided transportation support when needed or requested. Further, as in other research [47], we collected the adult participant's contact information as well as the contact information of close friends/relatives, in case we had difficulty contacting the adult at follow-up. However, these additional contacts also had issues related to telephone service.

Attrition is expected in most intervention studies, but it is particularly a common challenge when working with hard to reach populations [47]. This is due, in part, to these participants experiencing a high number of unexpected and adverse life events [48]. In addition, participants who experience poverty necessarily face an increased number of resourcerelated issues including accessibility and transportation, both of which were challenges in this study. We had an overall attrition rate of $19 \%$. However, for data analysis and reporting purposes, the attrition rate was $25 \%$, because the three intervention dyads in which the adolescent completed the workshop via telephone were not included in the final analyses. Based on a meta-analysis of studies focused on behavioral interventions related to adolescents and health, the average attrition rate was $14.7 \%$ when follow-up was conducted within 63-6 months [49]. In a 6-month, community health worker intervention with African American and Latino adults with type 2 diabetes, the overall attrition rate was approximately $18 \%$ at follow-up [50]. Research with low-income families of color, in particular, can experience higher rates of attrition [51]. We believe that our attrition rates were relatively low, given the population from which we sampled. We attribute our retention rates to our use of community recruiters and mixed approaches to maintaining contact with participants.

As recommended by other researchers [52, 53], it is important to have facilitators or interviewers that are relatable. In our case, we had an African American woman as facilitator for each workshop. However, it is important that researchers and practitioners match compatibility on a variety of factors including age, language, current-lived experience, etc. Based on feedback about facilitator characteristics from pilot workshop participants, it is possible that a younger female whose mother had cancer would have been a more potent messenger. In addition, one of the YouTube appeals featured a celebrity and was the only video in which the speaker expressly talked about his mother having cancer. The participants were better able to recall this particular video and the information it presented, suggesting that information with a more direct connection to the mother figure and/or a celebrity better resonated with adolescents. For the girls who did not share the message or information with her relative, most either simply forgot or other conditions in their life prevented them from talking to their relative. For some participants, their life was very busy; they had also much going on that took priority over talking to their relative.

The lessons learned from the recruitment and retention of dyads in this study has strong implications for future studies. Researchers need to keep in mind the time commitment and the flexibility required to do recruiting and scheduling, especially with populations who may be experiencing multiple stressors due to their daily battles surviving poverty [54]. Providing individual sessions, as opposed to group workshops, may provide a solution to scheduling problems. Another potential solution to scheduling challenges would be to implement the workshops in pre-established groups, i.e., in school and sports teams. The efficacy of these approaches warrants additional research.

There were several of limitations of the study that should be considered when interpreting the findings. There was a small sample size in the pilot. In fact, only four dyads participated in the pilot testing, which may have limited the extent to which the information about the workshop process generalized to the feasibility study. Participants were paid for their research participation, which may have influenced their likelihood to participate. Some may argue that the appeal of the $\$ 100$ may have encouraged them to share the message with their adult relative. However, those in the control group, who were not offered any additional incentive, also shared the screening message at equally high rates with their adult relative. Furthermore, it may be that this sample was bias, given that they knew they were recruited for a health communication study although they were not told it was for a cancer-related study. However, it could be that the survey questions could have alerted them to the fact that it was a cancer-related study. Nevertheless, this was a high-risk sample, in that these women did not comply with screening recommendations, and while the questions may have alerted them to the nature of the study, we are confident that these questions alone could not have activated them to obtain screening. An additional limitation was that we were unable to recruit an equal amount of dyads 
for our control group and thus were unable to make strong comparisons. In the future, implementation fidelity should be assessed. In addition, efforts to control the amount of time that lapses between baseline, intervention, and follow-up should be made. While no differences between mother-daughter dyads and adult relative-daughter dyads were apparent, the sample sizes were relatively small to determine significant differences. It may be that daughters communicate important messages differently to their adult relative than to their mother; however, regardless of caretaker role, the girls were successful at delivering the cancer message to the selected adult in their lives. This is an added contribution to the study, because it demonstrates that important messages can be successfully delivered outside of the home to other adults, and by utilizing this approach, we may be able to reach a wider population with important health information. Future studies could investigate to what extent differences in communication to mothers versus other female relatives exist and to what extent these differences influence outcomes.

Despite these limitations, the findings from this study have important implications for intergenerational and bidirectional health promotion knowledge. In general, we found that adolescents not only were capable of delivering an important health message to their relative but also can also deliver a successful screening appeal. However, researchers who wish to include mother-daughter dyads must take into consideration resources in time and money, as well as establishing a variety of strategies for recruitment, contact, involvement, and retention of participants. Recruiting mother-daughter dyads in populations disparately impacted by cancer is feasible, but the challenges addressed in this paper should be considered prior to intervention development and implementation.

Acknowledgments All procedures followed were in accordance with the ethical standards of the responsible committee on human experimentation. Informed consent was obtained from all participants for being included in the study.

Conflict of interest This research was supported by NIH/NCI K01 CA132960. No author has any conflict of interest.

\section{References}

1. Champion V et al. A tailored intervention for mammography among low-income African-American women. J Psychosoc Oncol. 2000;18(4):1-13.

2. American Cancer Society. Cancer facts \& figures for African Americans 2011-2012. Atlanta: American Cancer Society; 2012.

3. Ward $\mathrm{E}$ et al. Cancer disparities by race/ethnicity and socioeconomic status. CA Cancer J Clin. 2004;54(2):78-93.

4. Brandt HM et al. Relationship of colorectal cancer awareness and knowledge with colorectal cancer screening. Color Cancer. 2012;1(5):383-96.
5. Brown DR et al. Cervical cancer screening among ethnically diverse black women: knowledge, attitudes, beliefs, and practices. J Natl Med Assoc. 2011;103(8):719.

6. Robb $\mathrm{K}$ et al. Ethnic disparities in knowledge of cancer screening programmes in the UK. J Med Screen. 2010;17(3):125-31.

7. Paskett ED et al. Racial difference in knowledge, attitudes, and cancer screening practices among a triracial rural population. Cancer. 2004;101(11):2650-9.

8. Summers C et al. Racial/ethnic differences in early detection of breast cancer: a study of 250,985 cases from the California Cancer Registry. J Women's Health. 2010;19(2):203-7.

9. Barnholtz-Sloan J et al. Incidence trends of invasive cervical cancer in the United States by combined race and ethnicity. Cancer Causes Control. 2009;20(7):1129-38.

10. Jemal A et al. Cancer Statistics 2004. CA Cancer J Clin. 2004;54(1): 8-29.

11. Fowler B. Social processes used by African American women in making decisions about mammography screening. J Nurs Scholarsh. 2006;38(3):247-54.

12. Millon-Underwood S. Determinants of participation in state-of-theart cancer prevention, early detection/screening, and treatment trials among African-Americans. Cancer Nurs. 1993;16(1):25-33.

13. Giuliano AR et al. Participation of minorities in cancer research: the influence of structural, cultural, and linguistic factors. Ann Epidemiol. 2000;10(8, Supplement 1):S22-34.

14. Robinson JM, Trochim WMK. An examination of community members', researchers' and health professionals' perceptions of barriers to minority participation in medical research: an application of concept mapping. Ethn Health. 2007;12(5):521-39.

15. National Institutes of Health. Monitoring adherence to the NIH policy on the inclusion of women and minorities as subjects in clinical research. 2007. http://orwh.od.nih.gov/inclusion/FinalAnnualReport2006.pdf

16. Yancey AK, Ortega AN, Kumanyika SK. Effective recruitment and retention of minority research participants. Annu Rev Public Health. 2006;27(1):1-28.

17. Sheikh A. Why are ethnic minorities under-represented in US research studies? PLoS Med. 2006;3(2):e49.

18. Campbell LC, McClain J. Exploring prostate cancer literacy and family cancer awareness in college students: getting ahead of the curve in cancer education. J Cancer Educ. 2013;28(4):617-22.

19. Gaber $\mathrm{R}$ et al. Communication by mothers with breast cancer or melanoma with their children. Int J Environ Res Pub Health. 2013;10(8):3483-501.

20. Gursoy A et al. A different approach to breast self-examination education: daughters educating mothers creates positive results in Turkey. Cancer Nurs. 2009;32(2):127-34.

21. Browne JL, Chan AY. Mother-daughter communication about mammography in an Australian sample. J Fam Commun. 2012;12(2): 129-50.

22. Mosavel M. The feasibility of mothers accepting health advice from their adolescent daughters. J Health Care Poor Underserved. 2009;20(1):42-9.

23. U.S. Department of Health and Human Services - National Center for Research Resources. Women's health USA 2012. U.S. Department of Health and Human Services - Health Resources and Services Administration. Rockville, MD: Maternal and Child Health Bureau; 2013.

24. Hutchinson MK and TM Cooney. Patterns of parent-teen sexual risk communication: implications for intervention. Fam Relat. 1998:185-94.

25. Noller P, Bagi S. Parent-adolescent communication. J Adolesc. $1985 ; 8(2): 125-44$.

26. Youniss J and Smollar J. Adolescent relations with mothers, fathers and friends. University of Chicago Press; 1987.

27. Fingerman KL. "We had a nice little chat": age and generational differences in mothers' and daughters' descriptions of enjoyable visits. J Gerontol B Psychol Sci Soc Sci. 2000;55(2):P95-106. 
28. Mosavel M. Health promotion and cervical cancer in South Africa: why adolescent daughters can teach their mothers about early detection. Health Promot Int. 2012;27(2):157-66.

29. Jones FC, Broome ME. Focus groups with African American adolescents: enhancing recruitment and retention in intervention studies. J Pediatr Nurs. 2001;16(2):88-96.

30. Belgrave FZ. Relational theory and cultural enhancement interventions for African American adolescent girls. Public Health Rep. 2002;117 Suppl 1:S76.

31. Shields AM et al. Subject recruitment and retention against quadruple challenges in an intervention trial of end-of-life communication. $J$ Hosp Palliat Nurs. 2010;12(5):312-8.

32. Northouse LL et al. Research with families facing cancer: the challenges of accrual and retention. Res Nurs Health. 2006;29(3):199-211.

33. Sadler GR et al. Costs of recruiting couples to a clinical trial. Contemp Clin Trials. 2007;28(4):423-32.

34. Quinn $C$ et al. Challenges and strategies of dyad research: cardiovascular examples. Appl Nurs Res. 2010;23(2):e15-20.

35. Wilson MN. Child development in the context of the Black extended family. Am Psychol. 1989;44(2):380.

36. Burton LM. Intergenerational patterns of providing care in AfricanAmerican families with teenage childbearers: emergent patterns in an ethnographic study. In: Bengtson VL, Schaie KW, Burton LM, editors. Adult intergenerational relations: effects of social change. New York: Springer; 1995. p. 79-118.

37. Mosavel M, Genderson MW. From adolescent daughter to mother: exploring message design strategies for breast and cervical cancer prevention and screening. J Cancer Educ. 2013;28(3):558-64.

38. Petty RE, Cacioppo JT. Attitudes and persuasion - classic and contemporary approaches. Dubuque, IA: W.C. Brown; 1981. p. xvii. 314 p.

39. Harvey AR, Rauch JB. A comprehensive Afrocentric rites of passage program for black male adolescents. Health Soc Work. 1997;22(1): 30-7.

40. Ajzen I. The theory of planned behavior. Organ Behav Hum Decis Process. 1991;50(2):179-211.

41. Prather $\mathrm{C}$ et al. Diffusing an HIV prevention intervention for African American women: integrating afrocentric components into the SISTA diffusion strategy. AIDS Educ Prev. 2006;18(Suppl):149-60.
42. Ferguson SL. Peer counseling in a culturally specific adolescent pregnancy prevention rogram. J Health Care Poor Underserved. 1998;9(3):322-40.

43. Susan D et al. Peripheral cues and involvement level: influences on acceptance of a mammography message. J Health Commun. 1998;3(2):119-35.

44. Dixon AC, Schoonmaker CT, Philliber WW. A journey toward womanhood: effects of an Afrocentric approach to pregnancy prevention among African-American adolescent females. Adolescence. 2000;35(139):425-9.

45. Richman KA, Alexander LB, True G. Proximity, ethical dilemmas, and community research workers. AJOB Prim Res. 2012;3(4):19-29.

46. Dumka $L$ et al. Recruitment and retention of high-risk families into a preventive parent training intervention. J Prim Prev. 1997;18(1): $25-39$.

47. Lindenberg CS et al. Challenges and strategies for conducting intervention research with culturally diverse populations. J Transcult Nurs. 2001;12(2):132-9.

48. McLoyd VC. The impact of economic hardship on black families and children: psychological distress, parenting, and socioemotional development. Child Dev. 1990;61(2):311-46.

49. Robin $\mathrm{L}$ et al. Behavioral interventions to reduce incidence of HIV, STD, and pregnancy among adolescents: a decade in review. J Adolesc Health. 2004;34(1):3-26.

50. Spencer MS et al. Effectiveness of a community health worker intervention among African American and Latino adults with type 2 diabetes: a randomized controlled trial. Am J Public Health. 2011;101(12):2253-60.

51. Gross D, Julion W, Fogg L. What motivates participation and dropout among low-income urban families of color in a prevention intervention? Fam Relat. 2001;50(3):246-54.

52. Anderson RM et al. Using focus groups to identify psychosocial issues of urban black individuals with diabetes. Diabetes Educ. 1996;22(1):28-33.

53. Jackson JS. Life in black America. Sage Publications; 1991.

54. Murray DM et al. Enhancing cancer screening in primary care: rationale, design, analysis plan, and recruitment results. Contemp Clin Trials. 2013;34(2):356-63. 